\title{
Introduction - Les transversalités de l'agriculture bio
}

\author{
Michel Streith et Céline Cresson
}

\section{(2) OpenEdition \\ Journals}

\section{Édition électronique}

URL : http://journals.openedition.org/economierurale/4198

DOI : 10.4000/economierurale.4198

ISSN : 2105-2581

\section{Éditeur}

Société Française d'Économie Rurale (SFER)

\section{Édition imprimée}

Date de publication : 20 janvier 2014

Pagination : 6-8

ISSN : 0013-0559

\section{Référence électronique}

Michel Streith et Céline Cresson, «Introduction - Les transversalités de l'agriculture bio », Économie rurale [En ligne], 339-340 | janvier-mars 2014, mis en ligne le 20 janvier 2014, consulté le 04 mai 2019. URL : http://journals.openedition.org/economierurale/4198; DOI : 10.4000/economierurale.4198 


\title{
INTRODUCTION
}

\section{Les transversalités de l'agriculture bio}

\author{
Michel STREITH • CNRS \\ Céline CRESSON • ACTA
}

D ifférents événements autour de l'agriculture biologique $(A B)$ ont eu lieu ces dernières années, chacun abordant une ou plusieurs dimensions de cette activité. En 2008, le colloque DinABio est un moment clé par rapport à la dynamique de l'agriculture biologique et à la programmation d'engagements institutionnels précis en matière de recherche et de développement. Il fait le constat d'une évolution des sujets traités et mentionne quelques avancées significatives ${ }^{1}$. Au plan scientifique, il souligne la présence de démarches systémiques interdisciplinaires et la mobilisation croissante de l'INRA dans l'agriculture biologique tout en pointant la persistance de lacunes importantes qui subsistent et la nécessité d'explorer des pistes de recherche nouvelles ${ }^{2}$. Le RMT DévAB (Réseau mixte technologique pour le développement de l'agriculture biologique) organise, depuis 2008, de nombreux séminaires permettant aux acteurs de I'AB de se rencontrer avec pour objectifs de valoriser les travaux de recherche et de développement et de consolider de nouveaux partenariats dans la perspective de projets à élaborer, en France et en Europe. En juin 2011, la SFER (Société française d'économie rurale) et le RMT DévAB organisent un colloque sur les « Transversalités de l'agriculture biologique » qui se tient à la Maison interuniversitaire des sciences humaines d'Alsace, à Strasbourg ${ }^{3}$. Cette nouvelle étape dans le partage des connaissances est l'occasion de rencontres entre chercheurs et techniciens de différentes disciplines, entre institutions peu coutumières à échanger leurs savoirs et entre mondes encore trop cloisonnés. Les chapitres du numéro d'Économie rurale constituent à la fois un panorama des travaux qui se sont déroulés durant les journées du colloque et la mise en perspective des pistes de réflexion qu'il reste à défricher.

L'initiative s'appuie sur la logique des orientations et des changements qui traversent l'agriculture biologique depuis une dizaine d'années. La justification de l'engagement d'agriculteurs dans un mode de production en agriculture biologique repose en grande partie sur les dimensions transversales de cette activité. Les motivations obéissent généralement à plusieurs finalités ou conduites (agronomiques, environnementales, sociales, économiques, esthétiques, éthiques, émotionnelles, militantes) orientées selon des objectifs et des valeurs croisés. À l'échelle de la société, la montée en puissance de l'agriculture biologique correspond à un moment historique de

1. Innovations agronomiques, vol. 4, janvier 2009. http://www6.inra.fr/ciag/Revue/Volume-4-Janvier-2009.

2. Meynard J.-M. (2009). Conclusion : quelles priorités pour la R\&D en agriculture biologique ? Innovations agronomiques, vol. 4, p. 495-498.

3. Les communications du colloque peuvent être téléchargées gratuitement sur le site du RMT DévAB : www.devab.org/ moodle/course/view.php?id=43ou sur le site de la SFER : http://www.sfer.asso.fr/index. php/sfer_asso/les_colloques_thematiques/les_colloques_passes/les_transversalites_de_1_agriculture_ biologique/programme_actes_du_colloque. 
remise en question de modes de vie, de s'alimenter, de penser la relation à notre environnement et de produire autrement. Les pratiques techniques et sociales des agriculteurs bio traversent donc des territoires sociaux très divers, tant au plan individuel que collectif.

En dépit de l'intérêt scientifique de l'agriculture biologique, tant sur le plan des innovations agronomiques que sociales, la recherche n'a pas encore suffisamment pris en compte l'intérêt du phénomène $A B$ dans la construction de nouveaux outils méthodologiques et théoriques. Tout au plus assiste-t-on à des projets où émergent quelques tentatives de transdisciplinarité. Mais, nous restons insérés dans des dimensions organisationnelles où subsistent des hiérarchies d'expertise (dimension verticale) ou l'entre-soi disciplinaire (dimension horizontale).

Cet ouvrage, comme le colloque dont il est en partie issu, se veut une tentative de dépasser les cadres institutionnels et formels de la recherche sur l'agriculture biologique pour celui des relations humaines qui les composent. En cela, nous revendiquons la définition de la transversalité de Félix Guattari : "Mettez dans un champ clos des chevaux avec des œillères réglables et disons que le coefficient de transversalité sera justement ce réglage des œillères [...] Au fur et à mesure qu'on ouvrira les œillères, on peut imaginer que la circulation sera réalisée de façon plus harmonieuse ${ }^{4}$. " Ce fut la circulation des idées et des savoirs qui guida notre choix de faire se rencontrer des chercheurs, des experts et des disciplines qui travaillent sur le même sujet, mais ne se côtoient jamais ou très rarement. Certes, le projet est humble, mais il garantit une pratique scientifique apte à participer au développement de l'agriculture biologique.

Afin de partager nos savoirs sur les dimensions transversales de l'agriculture biologique, le colloque a été organisé à partir des thématiques suivantes : les nouveaux enjeux économiques, les pratiques et les discours, l'identité, les passerelles scientifiques.

Différents enjeux économiques ont été regardés et nous ont permis d'aborder des questions importantes. Concernant le volet agricole proprement dit, nous nous sommes interrogés sur l'économie de la production (la rentabilité des exploitations biologiques est-elle équivalente au conventionnel ?) et l'économie spatiale (comment les fermes biologiques se répartissent sur le territoire français ?). Du côté des consommateurs, nous avons étudié l'économie de l'environnement (en mettant en question l'empreinte écologique du consommateur de produits biologiques par rapport à d'autres), l'économie de la consommation (à quelles motivations obéissent les consommateurs de produits biologiques ?), et la théorie du comportement planifié (à partir du cas de la consommation de produits biologiques régionaux en Midi-Pyrénées). Nous avons abordé également des sujets plus « classiques » en économie. Ainsi en est-il de la régulation et de la gouvernance des filières (exemples d'organisation des coopératives pour structurer l'agriculture biologique dans des régions aux profils agronomiques différents), du rôle joué par les collectivités territoriales. Nous avons regardé aussi ce qui se passait pour l'agriculture biologique hors de France : insignifiante au Japon malgré une politique volontariste, stagnante en Allemagne en dépit de la présence de producteurs très organisés et de stratégies commerciales très actives, la production et la consommation de produits biologiques subit des fluctuations sous l'effet d'événements exogènes. L'exemple le plus caractéristique concerne le nombre croissant d'importations. Cet atelier est mis en évidence par les articles de Allaire et al., Petit et al. et Touret et al. qui conjuguent des approches territorialisées, à des échelles différentes, et des processus de développement économique de l'AB.

4. Guattari F. (1972). Psychanalyse et transversalité. Essai d'analyse institutionnelle. Paris, La Découverte, p. 79. 


\section{INTRODUCTION}

L'atelier consacré à la thématique " pratiques et discours " a rendu compte (i) des transformations et des dynamiques de l'agriculture biologique, (ii) d'expérimentations et d'innovants menés à des échelles micro, mais susceptibles d'être transposées à plus grande échelle, (iii) de la gestion rationnelle de l'agriculture biologique et de ses effets imprévus, (iv) de l'apprentissage et de l'organisation de formations. Ces thématiques avaient pour objectif commun de réfléchir aux processus de planification, d'improvisation et d'initiative afin de qualifier les performances et les fonctionnements de l'agriculture biologique. Les recherches présentées dans les différentes interventions se sont appuyées sur des données empiriques élaborées à partir de terrains ou de contextes comparatifs : bio/non bio, global/local, éthique/économique. Cet atelier est illustré par les articles d'Alavoine-Mornas et al., Coquil et al., Fleury et al. et Pernin et al.

L'atelier " identités " s'est structuré autour du domaine des sciences sociales et des communications. II porte des interrogations fortes par rapport à la question des recompositions à l'œuvre dans le champ de l'agriculture biologique et dans la redéfinition de ses frontières : champ en mouvement traversé par différentes tensions (constructions identitaires, oppositions, trajectoires de développement, spécificités des territoires, logiques différentes). II interroge les transversalités à l'œuvre à plusieurs échelles : celle des fermes (évaluation transversale des performances) où l'identité est abordée à partir d'individus ou de groupes sociaux, et celle des territoires (système d'acteurs territorialisés) avec une importance des approches qualitatives (étude de cas). Cet atelier a permis de montrer que l'agriculture biologique pousse le conventionnel à introduire une écologisation des pratiques, grâce à la multiplication des échanges entre agriculteurs et institutions du monde bio et non bio. Cela se traduit par un décloisonnement entre ces deux mondes, mais aussi par un repositionnement de l'agriculture biologique dans le champ agricole et une appropriation de l'AB par de nouveaux acteurs (citoyens, agents économiques, acteurs de la recherche). De même, nous assistons à une montée en puissance des politiques publiques territorialisées dans le développement du soutien à l'agriculture biologique. Néanmoins, nous constatons aussi le manque d'approches complémentaires entre amont et aval des filières, de développement local, d'intégration à des échelles supérieures, de constitution de réseaux. Ces thématiques sont abordées par les articles de Gafsi et al., Sautereau et al., Nizet et al. et Cardona.

L'atelier " passerelles scientifiques " a permis de mettre au jour la pluralité des visions de I'agriculture biologique dans les domaines de la recherche, de la formation et du développement, facteurs essentiels du devenir de ce système de production. II a également permis de renouveler nos catégories d'analyse et de rediscuter les frontières qui nous séparent, tant dans nos pratiques professionnelles que dans nos sujets de réflexion. Ce travail est nécessaire pour favoriser des passerelles ou des "fronts ", redéfinir des réseaux, avoir une réflexivité accrue sur les projets, équiper l'agriculture biologique et relier celle-ci à d'autres formes d'agriculture.

Refusant à tout optimisme unanime qui caractérise généralement le bilan d'un colloque ou d'une publication, nous faisons preuve de prudence. L'atelier " passerelles scientifiques » n'a pas pu faire l'objet d'un rendu scientifique en matière d'articles. Nous sommes là dans un domaine où tout est à construire. Pour cela, il convient de valoriser les compétences, d'instruire des controverses et ainsi d'optimiser la recherche et le développement en agriculture biologique. Les faits économiques, agronomiques et sociaux nous ont précédés. Ils sont le ferment augurant de pratiques scientifiques transversales qui restent à inventer. 